\title{
Enabling Access Through Real-Time Sign Language Communication Over Cell Phones
}

\author{
Jaehong Chon*, Neva Cherniavsky ${ }^{\dagger}$, Eve A. Riskin* and Richard E. Ladner ${ }^{\dagger}$ \\ *Department of Electrical Engineering, University of Washington, Seattle, WA 98195 \\ \{jaehong, riskin\}@u.washington.edu \\ ${ }^{\dagger}$ Department of Computer Science and Engineering, University of Washington, Seattle, WA 98195 \\ \{nchernia, ladner\}@cs.washington.edu
}

\begin{abstract}
The primary challenge to enabling real-time twoway video conferencing on a cell phone is overcoming the limited bandwidth, computation and power. The goal of the MobileASL project is to enable access for people who use American Sign Language (ASL) to an off-the-shelf mobile phone through the implementation of real-time mobile video communication. The enhancement of processor, bandwidth, and power efficiency is investigated through SIMD optimization; region-of-interest encoding based on skin detection; video resolution selection (used to determine the best trade off between frame rate and spatial resolution); and variable frame rates based on activity recognition. Our prototype system is able to compress, transmit, and decode 12-15 frames per second in real-time and produce intelligible ASL at $30 \mathrm{kbps}$. Furthermore, we can achieve up to 23 extra minutes of talk time, or a $8 \%$ gain over the battery life of the phone, through our frame dropping technique.
\end{abstract}

\section{INTRODUCTION}

Many issues have prevented real-time video communication on the mobile phone. Mobile video communication is already available in Japan and Europe, but regardless of the higher bandwidth $3 \mathrm{G}$ network there, the quality is poor, the videos are jerky, and there is significant delay. With the advent of mobile phone PDAs equipped with larger screens and photo/video capture, people who communicate with ASL should be able to use these new technologies.

Mobile phone service providers operate wireless networks using many different communication standards. The most widely used of these technologies is called the Global System for Mobile Communications, or GSM. On top of a GSM network, they operate a data network called the General Packet Radio Service (GPRS) and an upgrade for faster speeds called Enhanced Data Rates for GSM Evolution (EDGE), which can carry data speeds from $35.2 \mathrm{kbps}$ up to $236.8 \mathrm{kbps}$. Furthermore, they have launched a high-speed network based on UMTS and High-Speed Downlink Packet Access (HSDPA), which is commonly known as $3 \mathrm{G}$. Since a video call is bidirectional, the effective bandwidth is limited by the uplink. Our project, with its target bit rate of $30 \mathrm{kbps}$, is well suited to current U.S. cellular data networks.

The computing capability on mobile phones is very low because of low clock frequencies, single cores, and only basic Single Instruction Multiple Data (SIMD). In our work, we utilize assembly optimization to solve the issue of limited processing power. Second, we implement dynamic regionof-interest (ROI) coding based on skin detection to reduce the required bandwidth while keeping the amount of quality and intelligibility needed for ASL. Third, we investigate a variable frame rate to prolong the battery life time by taking advantage of the fact that ASL speakers usually sign when the other person isn't signing. Building on previous work[1], [2], we optimize a variable frame rate. Finally, we design ASL encoders that use $\mathrm{x} 264[3]$ at a high enough frame rate in real time on the mobile phone.

\section{BACKGROUND}

MobileASL[4] is a video compression project at the University of Washington and Cornell University[5] with the goal of making cell phone communication for people who use sign language a reality in the U.S. Our software runs on the Windows Mobile operating system. MobileASL is compatible with the H.264/AVC compression standard and can be decoded by any H.264 decoder. The x264 Open Source H.264 encoder was selected because of its fast speed[3], [6], [7], making it a good choice for compression on low-power devices such as mobile phones. In [6], x264 showed better quality than several commercial H.264/AVC encoders. When compared with the JM reference encoder (version 10.2)[8], x264 (version 0.47.534) was shown to be 50 times faster, while providing bit rates within $5 \%$, for the same PSNR.

\section{A. MobileASL System}

Since the Internet Protocol Suite (commonly known as $\mathrm{TCP} / \mathrm{IP}$ ) is used for the Internet and other similar networks, we designed a lightweight TCP/IP networking system to use the MobileASL codec on two different networks: the WiFi network and a current cellular data network in the U.S. (AT\&T).

In our system, we use a light and simple protocol over TCP for the call signaling, e.g., connection establishment, connection release, and connection control such as for packet loss indication. We use UDP for video transmission. Fig. 1 shows the TCP/IP layered architecture, which is assisted by an HTTP protocol for MobileASL.

The HTTP protocol is used to register user information (such as the IP address) at the server, which is located outside the network. Whenever the user calls another person, our system retrieves its IP address from the server and uses it to make a connection. 


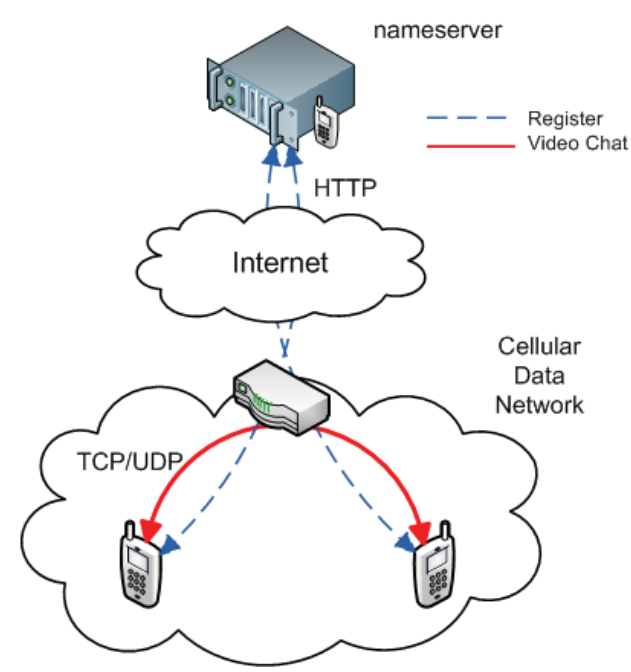

Fig. 1. Network Architecture for MobileASL.

\section{B. Hardware Platform}

We use the HTC TyTN-II mobile phone which has a Qualcomm MSM7200 (400MHz ARM ARM1136EJ-S processor). We chose this phone because it has a front camera on the same side as the screen and runs Windows Mobile 6.1. Its processor adopts the ARMv6 core having a new SIMD instruction set[9]. This phone has a 320x240 pixel screen, a VGA video call front camera, a QWERTY keyboard, a H.264 hardware decoder, etc. Also, the HTC TyTN-II provides wireless capabilities such as Wi-Fi 802.11b/g, 2G (GPRS and EDGE) and 3G (HSDPA). Fig. 2 shows a MobileASL screenshot.

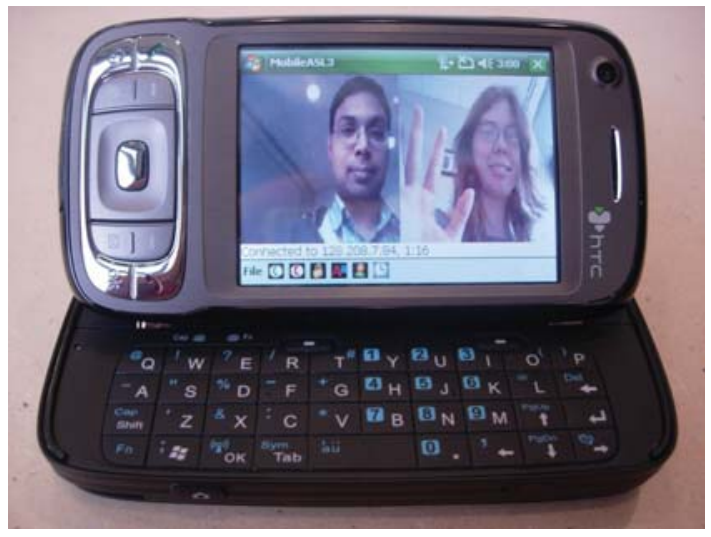

Fig. 2. A screenshot of our MobileASL codec on the HTC TyTN-II. Two UW graduate students are talking each other.

\section{SPEEDING UP The ENCODER}

Frame rates as low as 6 frames per second can be intelligible for signing though it would require the user to sign very slowly. A more comfortable frame rate would be 12 frames/second[10], and higher frame rates would be needed for fingerspelling[11], [12], [13]. To approach our desired frame rate of 12-15 frames/second, it was necessary to optimize the steps of the H.264 compression algorithm for motion estimation, mode decision, transforms, quantization and motion compensation using SIMD instruction sets. Then we determined the H.264 parameter settings and the video resolution needed to achieve our target frame rate.

\section{A. Assembly Optimization}

The ARM1136J-S processor used in the HTC TyTN-II is built around the ARM11 core in an integer unit that implements the ARM architecture v6. It supports a range of SIMD DSP instructions that operate on pairs of 16-bit values held in a single register, or on quadruplets of 8-bit values held in a single register[9]. The main operations supplied are addition, subtraction, multiplication, selection, pack and saturation. Operations are performed in parallel.

1) Motion Estimation: Motion estimation is the most timeconsuming module in video coding. The sum of absolute difference (SAD) is used as the distortion measure. In the ARMv6, the instruction USADA8 performs the sum of absolute differences of four 8-bit data and accumulates the results.

In H.264, there are 7 different block size types (from $16 \times 16$ to $4 \times 4)$. In the $16 \times 16,16 \times 8,8 \times 16,8 \times 8$ and $4 \times 8$ modes, there are 8 pixels in one line which are stored consecutively in memory. Using doubleword loading and USADA8, only 4 cycles are needed (2 loads and 2 SAD operations). Fig. 3 shows this process.

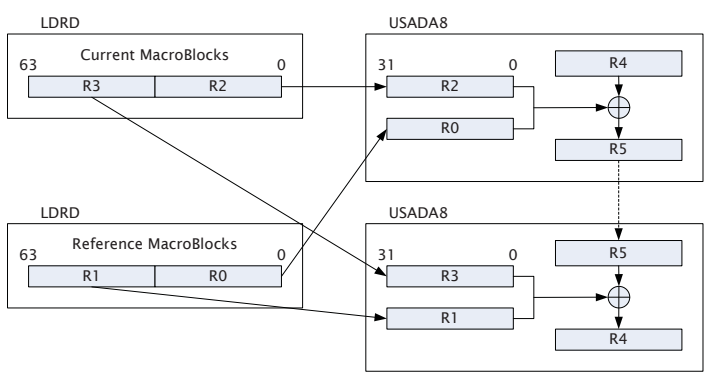

Fig. 3. SAD calculation using the USADA8 instruction.

In the $8 \times 4$ and $4 \times 4$ modes, one line has 4 consecutive pixels (32 bits), so we can load two lines of data to the register with load instruction and then they are calculated with USADA8. This costs 3 cycles. For the best optimization results, we use one of two methods depending on what mode is selected.

2) Mode Decision: The H.264 encoder uses rate-distortion optimization to select the mode. Because of its complexity, mode selection is clearly an important candidate for speed up.

The distortion is computed as the sum of squared differences (SSD) between the original block and the reconstructed block. The SSD for 4 pixels between two macroblocks can be optimized using three SIMD instructions. There are two consecutive steps which are 8-bit absolute difference (UQSUB8 and ORR) and then multiplication (SMLAD). It enables us to get approximately twice the speed otherwise possible.

3) Transforms: H.264 uses three transforms depending on the type of residual data that are to be coded: a transform for the $4 \times 4$ array of luma DC coefficients in intra macroblocks 


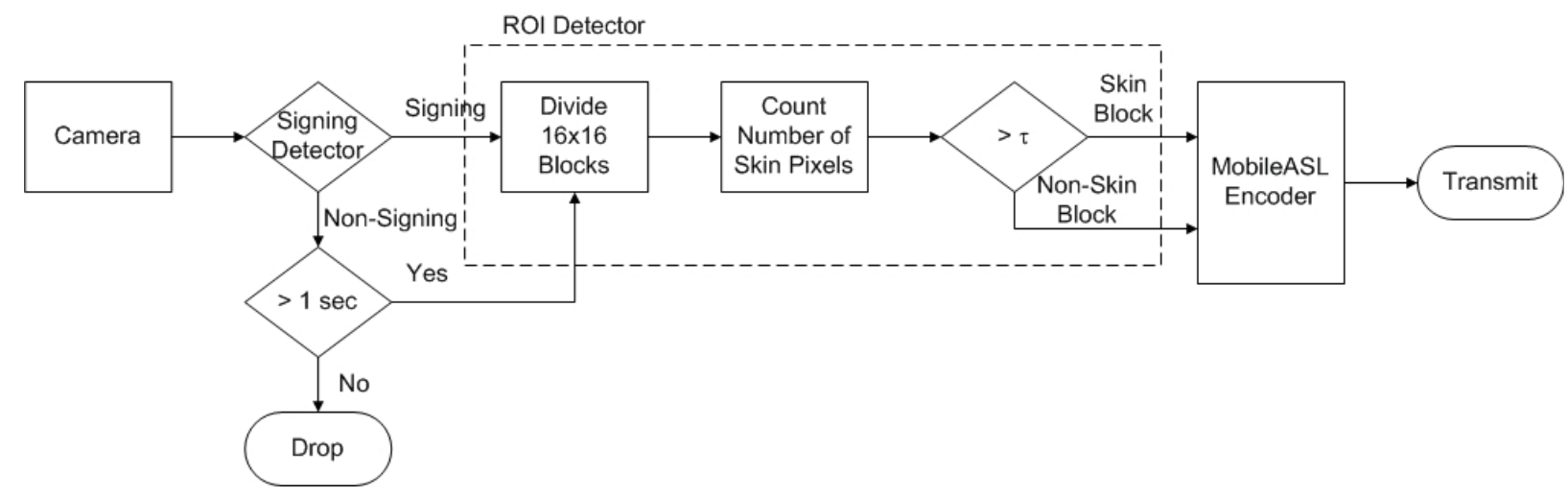

Fig. 4. MobileASL Framework. The variable frame rate and ROI detector based on skin blocks are concatenated before encoding.

(predicted in $16 \times 16$ mode); a transform for the $2 \times 2$ array of chroma DC coefficients (in any macroblock); and a transform for all other $4 \times 4$ blocks of residual data.

The DC coefficient of each $4 \times 4$ block in the $16 \times 16$ Intra prediction mode is transformed using a twice 1-D $4 \times 4$ Hadamard transform, which uses additions and subtractions (QADDSUBX, QADD16, QSUB16) and shifts (SHADD16) in 16-bit arithmetic. The other two transforms are also implemented in a similar way.

\section{B. H.264 Parameters Optimization}

After we optimized the encoder in assembly, we determined H.264 parameter settings that have low complexity but still offer high video quality at $30 \mathrm{kbps}$ by training on 6 videos recorded with a cell phone at QCIF resolution. We found that setting some x264 encoding parameters, such as the number of reference frames and motion search method, to their lowest complexity settings gave us almost the same quality as that of the highest complexity settings. Since the sub-pixel motion estimation parameter is highly complex, we chose its lowest complexity setting. We also chose $\mathrm{I} 4 \mathrm{x} 4, \mathrm{P} 8 \mathrm{x} 8$ for the partition size because it provides the best tradeoff of speed and quality.

\section{Video Resolution}

Even though we sped up the processing time through assembly and parameter optimization, we did not reach our target frame rate. Since H.264/AVC uses block-based motion compensation, the encoding time is highly related to the spatial resolution of the video. We investigate tradeoffs in spatial resolution versus speed for QCIF $(176 \times 144), 160 \times 128$, $144 \times 112,128 \times 96,112 \times 80,96 \times 80,80 \times 64,64 \times 48,48 \times 32$, and $32 \times 16$. In future work, we will conduct a controlled user study with ASL speakers to determine preferences for tradeoffs between spatial and temporal resolution.

\section{BANDWIDTH AND Power ENHANCEMENT}

Fig. 4 shows the MobileASL framework.

\section{A. Bandwidth Enhancement with ROI Encoder}

In [14], we used a fixed ROI by varying the level of distortion in a fixed region surrounding the face of the signer. We found that a tradeoff of 6 decreased quantization steps near the face of the signer (doubling the quality in that region) was preferred over a typical (no region-of-interest) encoding. We then extended this enhancement by finding face and hands regions using skin detection.

We first divide 16x16 blocks in each frames and then detect skin in real-time via a simple and well-known RGB-based algorithm[15] that works for many different skin tones. We designate macroblocks that have more pixels than a threshold as skin blocks. These blocks are encoded with lower quantization parameters to increase the quality. Fig. 5 shows which areas are considered to be skin blocks and how the quality of those blocks is enhanced.

\section{B. Power Enhancements}

Minimizing power consumption in mobile devices is an important challenge. We decrease frame rate during listening, which is equivalent to a voice codec that changes its own code rates based on the loudness or rhythm of speech in cellular system. Since turns are taken while speaking sign language, we can reduce the frame rate during the time of listening so that the usage of the encoder and wireless link will be decreased. This, in turn, decreases the amount of battery consumption.

In [1], we performed a user study to determine if variable frame rates sacrifice intelligibility and if automatic activity analysis is feasible. We used simple pixel differencing for speed.

1) Pixel Differences: For each frame $k$ in the video, we calculate the sum of absolute differences, $d(k)$, for each between the current and previous frames pixel. If it is greater than the threshold, $\tau$, we classify the frame as signing. We used ARMv6 SIMD instruction USADA8 to calculate the differences on the phone.

2) Signing vs. Not-Signing: We reduce the frame rate to 1 fps when the user is not-signing. To reduce false negatives, that is, signing frames being misclassified as not-signing, we only change to not-signing mode when three consecutive frames 

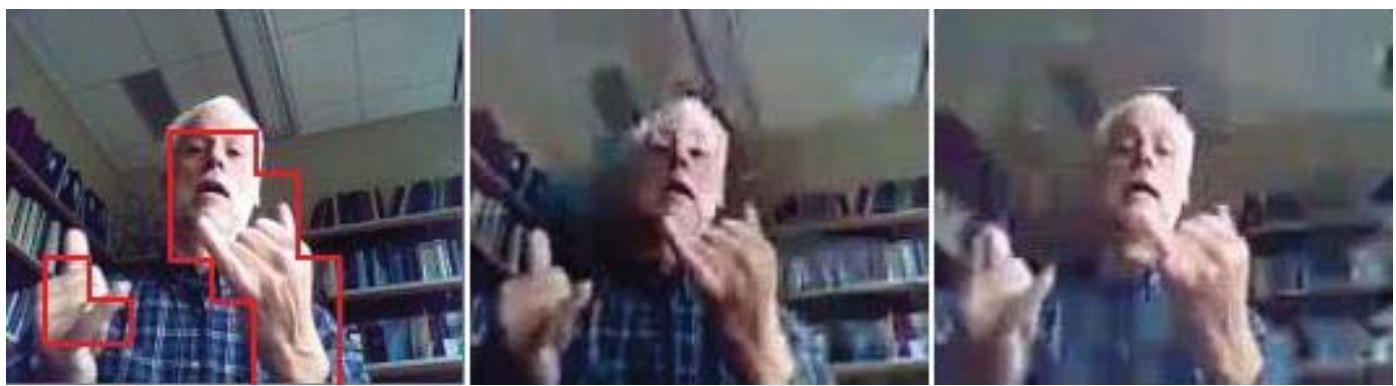

Fig. 5. A snap shot of ROI-based Encoder (a) original frame with skin blocks (red lines) (b) 0 ROI (c) 12 ROI. The quality in skin blocks is enhanced and quality in the rest of blocks is degraded.

TABLE I

Frame Per SECONd COMPARISON With MPEG AND ASL SeT (ENCODER ONLY).

\begin{tabular}{cccc}
\hline Type & QCIF test sequence & without SIMD (fps) & with SIMD (fps) \\
\hline \multirow{4}{*}{ ASL } & accident & 13.6 & 15.2 \\
& day in the life & 13.1 & 14.7 \\
& education & 14.6 & 16.1 \\
& favorite restaurant & 12.2 & 13.8 \\
& food at home & 12.6 & 14.3 \\
& graduation & 14.6 & 16.2 \\
& segment8 & 14.3 & 15.9 \\
\hline \multirow{4}{*}{ MPEG } & foreman & 9.7 & 11.2 \\
& carphone & 9.8 & 11.3 \\
& container & 11.8 & 13.2 \\
& grandma & 12.9 & 14.4 \\
& missam & 10.2 & 11.5 \\
& salesman & 14.7 & 16.3 \\
& akiyo & 14.6 & 16.1 \\
\hline
\end{tabular}

are detected as not-signing. In contrast, whenever we detect signing, we return immediately back to signing mode.

\section{EXPERIMENTAL RESULTS}

We present experimental results. First, we show the increase in speed achieved with SIMD optimization. Next, we describe how we not only reduced bandwidth but also achieved speed enhancement through ROI, followed by a description of battery life extension by frame dropping.

\section{A. SIMD Performance Comparison}

Experiments were conducted with fourteen QCIF $(176 \times$ 144) test sequences, each representing a different class of spatial detail and motion from the standard MPEG data set and an ASL data set developed at the University of Washington[16]. The MPEG set videos are foreman, carphone, container, grandma, missam, salesman and Akiyo and the ASL videos are accident, day in the life, education, favorite restaurant, food at home, graduation and segment8.

Table I shows a frames/second comparison for the encoder only for our data sets for two scenarios. Our instruction optimization for H.264 increased encoding frame rate up to $15.3 \%$ for the MPEG data set and $13.4 \%$ for the ASL data set.
TABLE II

PSNR COMPARISON OF DIFFERENT ROI.

\begin{tabular}{cccc}
\hline & 30kbps & 30kbps & 44kbps \\
& 0 ROI $(\mathrm{dB})$ & 12 ROI $(\mathrm{dB})$ & 0 ROI $(\mathrm{dB})$ \\
\hline ROI PSNR & 27.5 & 29.8 & 29.8 \\
Non-ROI PSNR & 29.3 & 24.3 & 31.4 \\
\hline
\end{tabular}

TABLE III

MACROBLOCK DISTRIBUTION IN P FRAMES FOR DIFFERENT ROI.

\begin{tabular}{ccc}
\hline Macroblock Size & 0 ROI (\%) & 12 ROI (\%) \\
\hline SKIP & 45.6 & 57.7 \\
Others & 54.4 & 42.3 \\
\hline
\end{tabular}

\section{B. ROI Encoding}

We compared the quality of the video recorded by the phone for three different ROIs $(0,6$ and 12$)$. Table II shows the PSNR for two different ROIs. The ROI PSNR for $12 \mathrm{ROI}$ at $30 \mathrm{kbps}$ corresponds to ROI PSNR for 0 ROI at $44 \mathrm{kbps}$. Therefore, we achieved a $32 \%$ bandwidth enhancement. As expected, 12 ROI increased quality in the ROI at the expense of quality in the background.

Another advantage of ROI-based encoding is its speed. Table III explains how macroblocks are selected for different ROIs during the mode decision stage of encoding. More bits are allocated to skin blocks and fewer bits are used for other blocks, more meaning blocks are chosen as skip blocks. The increased number of skip blocks also speed up macroblock encoding, entropy coding and slice coding (see Table IV). We speed up encoding by an additional $9.7 \%$.

Finally, the encoder is not the only module that consumes resources in real-time video communication. The decoder, the camera interface capturing the video, the screen interface displaying the video, and the transmission module all operate

TABLE IV

ENCODING TIME BREAKDOWN AND COMPARISON FOR DIFFERENT ROI.

\begin{tabular}{ccc}
\hline Encoder Function & 0 ROI (ms) & 12 ROI (ms) \\
\hline Mode Decision & 37.3 & 32.5 \\
Transform and Encoding & 12.2 & 10.0 \\
Entropy Coding (CAVLC) & 2.0 & 1.7 \\
NAL and Slice Coding & 2.7 & 2.6 \\
Others & 27.6 & 27.1 \\
\hline Total & 81.9 & 74.0 \\
\hline
\end{tabular}


simultaneously with the encoder. With our optimizations, our encoder occupies about $50 \%$ of the CPU time. As a result, we are able to achieve a frame rate of $12-15 \mathrm{fps}$ at $30 \mathrm{kbps}$ in real-time on the HTC TyTN-II. We demonstrated the success of our system over Wi-Fi and the AT\&T cellular network. A sample encoded ASL video can be viewed on YouTube[17].

\section{Picking The Resolution}

We performed the encoding of different video resolutions for the test sequences foreman and salesman from the MPEG data set and accident and graduation from our ASL data set. Based on these data, we chose the $96 \times 80$ resolution and can achieve up to $15 \mathrm{fps}$.

\section{Power Enhancement}

We simulated sign language communication and measured the instantaneous power usage every 5 seconds for an hour on the phones with the variable frame rate on and off. The power draw drops when the frame rate is lowered due to the lower processing power required to encode and transmit at 1 fps. Power saving is significant by utilizing frame dropping. This corresponds to 23 extra minutes of talk time, or a $8 \%$ power gain over the battery life of the phone (see Fig. 6).

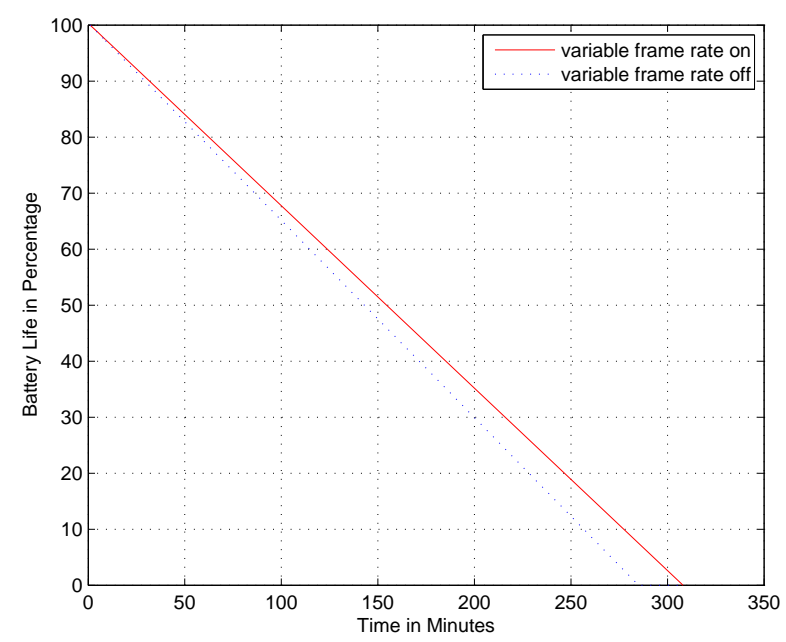

Fig. 6. Comparison of battery drain when encoding the video with the variable frame rate turned on.

\section{CONCLUSION}

In this paper, assembly optimization and parameter selection for an H.264 encoder are presented to enable real time video communication over a mobile phone. Experimental results demonstrate that we can provide 12-15 frames/second at a $96 \times 80$ resolution with our optimized ASL encoder. Our MobileASL framework with variable frame rate and ROI encoder is suitable for video communication in very low bit rate wireless network environments.

\section{ACKNOWLEDGMENT}

We would like to thank Louis Hsu and Fish Weng of HTC for helping us to get access to the front camera on the HTC TyTN-II cell phone. We also thank Jessica Tran for helping us measure battery drain on the cell phone. This work was supported by NSF grants CCF-0514353 and IIS-0811884.

\section{REFERENCES}

[1] N. Cherniavsky, A. Cavender, E. Riskin, and R. Ladner, "Variable Frame Rate for Low Power Mobile Sign Language Communication," in Proceedings of ASSETS 2007. Tempe, AZ, October 2007, pp. 163170.

[2] N. Cherniavsky, R. Ladner, and E. Riskin, "Activity Detection in Conversational Sign Language Video for Mobile Telecommunication," in Proceedings of 8th IEEE International Conference on Automatic Face and Gesture Recognition. Amsterdam, The Netherlands, September 2008.

[3] "x264 - a free H.264/AVC encoder," http://www.videolan.org/ developers/x264.html.

[4] "MobileASL," http://mobileasl.cs.washington.edu/.

[5] "Visual Communications Lab," http://foulard.ece.cornell.edu/.

[6] L. Merritt and R. Vanam, "Improved rate control and motion estimation for H.264 encoder," in Proceedings of ICIP, vol. 5, 2007, pp. 309-312.

[7] "4th Annual MSU MPEG-4 AVC/H.264 Video Codec Comparison,” 2007, http://www.compression.ru/video/codec_comparison/ mpeg-4_avc_h264_2007_en.html.

[8] "H.264/AVC JM Reference Software," http://iphome.hhi.de/suehring/ $\mathrm{tml} /$.

[9] “ARMv6-M Architecture Reference Manual," http://infocenter.arm.com/ help/index.jsp.

[10] I. T. S. Sector, "Draft application profile: Sign language and lip reading real time conversation usage of low bit rate video communication," 1998.

[11] R. A. Foulds, "Biomechanical and perceptual constraints on the bandwidth requirements of sign language," in IEEE Trans. On Neural Systems and Rehabilitation Engineering, vol. 12, March 2004, pp. Vol I: 65-72.

[12] G. Sperling, M. Landy, Y. Cohen, and M. Pavel, "Intelligible encoding of ASL image sequences at extremely low information rates," Computer vision, graphics, and image processing, vol. 31, no. 3, pp. 335-391, September 1985.

[13] B. F. Johnson and J. K. Caird, "The effect of frame rate and video information redundancy on the perceptual learning of American Sign Language gestures," in CHI '96: Conference companion on Human factors in computing systems. New York, NY, USA: ACM Press, 1996, pp. 121-122.

[14] A. Cavender, R. Vanam, D. Barney, R. Ladner, and E. Riskin, "MobileASL: Intelligibility of sign language video over mobile phones," in Disability and Rehabilitation: Assistive Technology. London: Taylor and Francis, June 2007.

[15] S. L. Phung, A. Bouzerdoum, and D. Chai, "Skin Segmentation Using Color Pixel Classification: Analysis and Comparison," IEEE Transactions on Pattern Analysis and Machine Intelligence, vol. 27, pp. 148154, January 2005.

[16] "MobileASL Study Videos," http://mobileasl.cs.washington.edu/ downloads/studyVideos/.

[17] "mobileasl-researchmovie," http://www.youtube.com/watch?v= FaE1PvJwI8E. 